\title{
Lupus and Pregnancy-15 Years of Experience in a Tertiary Center
}

\author{
Paula Ambrósio • Rita Lermann - Alexandra Cordeiro • \\ Augusta Borges • Isabel Nogueira • Fátima Serrano
}

Published online: 27 June 2009

(C) Humana Press Inc. 2009

\begin{abstract}
This retrospective study was designed to evaluate the outcome of pregnancies in women diagnosed with systemic lupus erythematosus (SLE) followed in a tertiary fetal-maternal center. Data were collected from clinical charts between January 1993 and December 2007, with a total of 136 pregnancies (107 patients). Mean maternal age was 29 years, with the vast majority of patients being Caucasian. Most patients were in remission 6 months prior to pregnancy (93\%) and the most frequently affected organs were the skin and joints. Renal lupus accounted for $14 \%$ of all cases. Twenty-nine percent of patients were positive for at least one antiphospholid antibody (aPL) and nearly 50\% had positive $\mathrm{SSa} / \mathrm{SSb}$ antibodies. All patients with positive aPL received low-dosage aspirin and lowmolecular-weight heparin (LMWH). There were no pregnancy complications in more than $50 \%$ of cases and hypertensive disease and intrauterine growth restriction were the most common adverse events. There were 125 live births, one neonatal death, eight miscarriages, and three medical terminations of pregnancy. Preterm delivery occurred in $25 \%$ of pregnancies. Our results are
\end{abstract}

P. Ambrósio $(\bowtie) \cdot$ R. Lermann · A. Cordeiro · I. Nogueira •

F. Serrano

Department of Fetal-Maternal Medicine,

Maternidade Dr. Alfredo da Costa,

Lisbon, Portugal

e-mail: paulaambrosio@netcabo.pt

A. Borges

Department of Medicine,

Maternidade Dr. Alfredo da Costa, Rua Viriato,

1069-089 Lisbon, Portugal

F. Serrano

Faculdade Ciências Médicas, Universidade Nova de Lisboa,

Maternidade Dr. Alfredo da Costa, Rua Viriato,

1069-089 Lisbon, Portugal probably the conjoined result of a multidisciplinary approach together with a systematic management of SLE pregnancies, with most patients keeping their prior SLE medication combined with low-dosage aspirin and LMWH in the presence of aPL.

Keywords Systemic lupus erythematosus · Pregnancy · Antiphospholipid antibodies

\section{Introduction}

Systemic lupus erythematosus (SLE) is a multisystemic disease predominantly affecting women in their reproductive years. It affects one in every 700 women with a peak incidence at around age 30 [1]. As the treatment of SLE improves, more women with this condition are able to become pregnant and the pregnancy loss rate has fallen from $43 \%$ in the 1960 s to $17 \%$ by 2000 [2]. Women with SLE have complicated pregnancies, with higher rates of fetal demise, hypertensive disease, thromboembolism, fetal growth restriction (FGR), premature delivery and neonatal death. Many of these complications can be attributed to SLE treatment more than to the disease itself. Several factors are believed to influence the outcome of pregnancy, namely lupus activity, antiphospholipid antibodies, renal status, hypertension and medication complications [3-5].

Antiphospholipid antibodies (aPL) are associated with arterial and venous thrombosis, pregnancy loss and thrombocytopenia although the presence of these antibodies per se has not been conclusively shown to be causal in thrombosis and miscarriage [6,7]. These antibodies are present in one third of patients with SLE (currently one diagnostic criteria according to the American College of Rheumatology), and its presence is associated with worse pregnancy outcome in SLE patients [5]. 


\section{Materials and methods}

Patients

Between 1993 and 2007, 107 women diagnosed with SLE (106 prior to pregnancy and one patient diagnosed during pregnancy) were followed at our autoimmune pregnancy clinic, accounting for 136 lupus pregnancies.

Pregnancies electively terminated for reasons other than medical were excluded from the study.

Methods

Retrospective study based on clinical charts assessing demographic, clinical, laboratory, therapeutic, pregnancy, and neonatal data. All data were submitted to descriptive summary statistical analysis.

Secondary antiphospholipid syndrome was diagnosed according to the Sapporo criteria [8].

The following definitions were used:

Nullipara: a woman who has never completed a pregnancy beyond an abortion

Multipara: a woman who has completed two or more pregnancies to viability

Pregnancy loss is defined as any pregnancy that does not result in a live infant beyond 28 days. A miscarriage is a spontaneous pregnancy loss before 20 weeks gestation and stillbirth is a pregnancy loss after 20 weeks gestation.

Neonatal death: death of a live-born infant within 28 days of birth

Preterm delivery: a birth which takes place after at least 20 , but less than 37, completed weeks of gestation related to last menstrual period (LMP)

Fetal growth restriction (FGR): fetal weight below the tenth percentile for gestational age.

Oligohydramnios: amniotic fluid index $<5$

Hypertension was defined as a systolic blood pressure $\geq 140 \mathrm{mmHg}$ and/or diastolic blood pressure $\geq 90 \mathrm{mmHg}$.

Thrombocytopenia was defined as a platelet count below $150,000 / \mathrm{mm}^{3}$

\section{Pregnancy management}

Patients were regularly examined according to obstetric and SLE evolution. At each visit, after vital signs assessment, every patient was submitted to a dipstick urine test and, if positive for proteinuria, to a 24 -h urine collection for total protein measurement.

Blood tests included standard obstetric evaluation with special attention to renal, hepatic and coagulation parameters. All patients were screened for the presence of aPL (lupus anticoagulant, anticardiolipin antibodies, beta-2-glycoprotein I).

Obstetric evaluation with standard sonographic evaluation plus additional uterine and umbilical Doppler flow was performed at 22, 28, and 32 weeks gestation and whenever indicated. Fetal echocardiogram was performed at 24 weeks gestation in patients with $\mathrm{SSa} /$ SSb-positive antibodies.

Patients were instructed to keep their SLE-specific medication (corticosteroids, hydroxychloroquine, azatioprin, cyclosporine) throughout pregnancy.

All women presenting with positive aPL, even in the absence of clinical criteria for secondary antiphospholipid syndrome, received low-dosage aspirin and lowmolecular-weight heparin after confirmation of fetal heart activity by fetal scan.

\section{Results}

\section{Maternal}

During the study period, there were 100,200 deliveries in Dr. Alfredo da Costa Maternity, of which 136 were lupus pregnancies followed in our clinic. Only one diagnosis was made during pregnancy, with all the others already studied and managed in rheumatology, nephrology, and internal medicine clinics from other centers before pregnancy.

Patients were mainly referred by other hospitals from the National Health System (84\%) and, in 5\% of cases, from private clinics; referral data were missing in $11 \%$ of cases.

Mean maternal age was 29 years and most women were nulliparous (Table 1).

Seventy-eight percent of women first attended our clinic during the first trimester $(<14$ weeks gestation) and $25 \%$ had a pre-conceptional visit. Mean gestational age at first visit was 11 weeks.

Table 1 Maternal characteristics

\begin{tabular}{lr}
\hline Maternal age (years) & \\
Mean & 29 \\
Max & 40 \\
Min & 19 \\
Ethnicity (\%) & \\
Caucasian & 95 \\
African & 5 \\
Parity (\%) & \\
Nulliparous & 62 \\
Multiparous & 38 \\
\hline
\end{tabular}


The most frequent comorbid illness in women with SLE was chronic hypertension ( $4 \%$ cases).

In terms of disease characteristics, articular and skin involvement accounted for the majority of cases, followed by kidney, blood, central nervous system, and serosal manifestations (Table 2). A high proportion of women were in remission for more than 6 months previous to pregnancy (93\%) and as for their immunologic profile, antiphospholipid antibodies (lupus anticoagulant, anticardiolipin antibodies, beta-2-glycoprotein I) were positive (at least one) in $29 \%$ of women with nearly $50 \%$ of patients testing positive for anti $\mathrm{SSa} / \mathrm{SSb}$ antibodies.

Eighty-six percent of patients received SLE-specific medication (mainly corticosteroids, hydroxychloroquine, and azatioprin); 4\% discontinued medication on their own initiative during pregnancy; the remaining were related to cutaneous lupus without treatment.

Pregnancy outcome

\section{Maternal}

There were no complications in more than half the pregnancies (52\% of cases) (Table 3 ). Nineteen patients developed hypertensive disease, with three cases of HELLP syndrome (hemolysis, elevated liver enzymes, low platelets). Lupus flares occurred in fivr patients during pregnancy (four renal and one articular). The articular lupus flare occurred after discontinuation of therapy and in all but one case of renal flare, the patients were in

Table 2 SLE characteristics

\begin{tabular}{lc}
\hline Disease duration (years) & 6.3 \\
Mean & 28 \\
Max & 0.3 \\
Min & \\
Disease status (\%) & 93 \\
Remission & 7 \\
Active disease & \\
Organ involvement (\%) & 54 \\
Articular & 53 \\
Skin & 28 \\
Kidney & 20 \\
Blood & 8 \\
CNS & 9 \\
Serosal & \\
Immunologic profile (\%) & \\
aPL & 29 \\
SSa/SSb & 49
\end{tabular}

$a P L$ antiphospholipid antibodies
Table 3 Pregnancy complications

\begin{tabular}{lr}
\hline Complications & $\%$ \\
No complications & 52 \\
Hypertensive complications & 14 \\
FGR & 14 \\
Flares & 4 \\
Fetal death (<20 weeks) & 8 \\
Oligohydramnios & 3 \\
Others & 5
\end{tabular}

FGR fetal growth restriction

remission for more than 6 months; all renal lupus cases kept their medication.

There were no cases of maternal death

\section{Delivery}

Ninety-four deliveries occurred after 37 weeks, with a preterm delivery rate of $25 \%$ (Table 4 ). Mean gestational age was 36.8 weeks (min-27 weeks; max - 40 weeks). Most patients had a vaginal delivery (55\%) and cesarean section was performed in 61 cases mainly for fetal distress.

\section{Fetal}

There were 125 deliveries (121 singleton and four twin pregnancies), one neonatal death, eight miscarriages before 12 weeks of gestation, and three medical terminations of pregnancy before 20 weeks of gestation. The latter were all for severe oligohydramnios (two renal and one articular lupus).

Fetal growth restriction occurred in $14 \%$ of cases with no obvious relationship with SLE activity or the presence of aPL.

Table 4 Fetal and delivery outcome

Fetal $(n)$

Live births

129

Neonatal death

1

Stillbirth

0

Miscarriage

8

TOP

3

Delivery $(n)$

Preterm

$31(25 \%)$

Term

$94(75 \%)$

Gestational age at delivery (weeks)

Mean

Max

40

Min

28

TOP medical termination of pregnancy

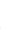

\section{.} . , .

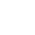
(n) (1) 
There were no cases of stillbirth.

The neonatal death was a baby delivered at 39 weeks by cesarean section for fetal distress with an APGAR score of $0 / 2$ at the first and fifth minute, respectively, that died on the 13th day with the diagnosis of severe asphyxia and grade 3 hypoxic-ischemic encephalopathy. Fetal weight at birth was 3,648 g. The mother was a 36-year-old nulliparous with cutaneous lupus with 4 years duration and in remission for more than 6 months prior to pregnancy. She was negative for aPL antibodies and was medicated with prednisone $5 \mathrm{mg}$ /day prior to pregnancy, which was unchanged during gestation. She developed gestational diabetes on the third trimester, managed with diet only and had no other co-morbidities. There were no post-partum complications, namely flares.

\section{Newborns}

Seventy-five percent of newborns had a birth weight above $2,500 \mathrm{~g}$, with $20 \%$ low-weight babies $(<2,500 \mathrm{~g})$ and $5 \%$ of very-low-weight babies $(<1,500 \mathrm{~g})$.

There were two cases of neonatal asphyxia.

There were no cases of neonatal lupus or congenital heart block.

\section{Discussion}

Systemic lupus erythematosus $<<<$ (SLE) is a complex chronic inflammatory disease that affects multiple organ systems and is clinically characterized by periods of remission and relapse [9]. The disease predominantly affects women and the incidence peaks between ages 15 and 45 years, i.e., the childbearing years, when the female-to-male ratio is about 12:1 .

Whether SLE activity increases during pregnancy has been a matter of debate over the last years although recent studies have found a two- to threefold increase in SLE activity during pregnancy [4, 10-12]. Differentiation of signs and symptoms of normal pregnancy from those of exacerbations of lupus (e.g. chloasma vs. malar rash, proteinuria secondary to preeclampsia vs. proteinuria due to lupus nephritis) can be particularly difficult, which clearly constitutes a bias when assessing reported incidence and prevalence rates. On the other hand, in the past, many patients used to stop all their therapy upon discovering that they were pregnant which may have contributed to an increased risk of flare in pregnancy $[10,11,13]$.

When compared with other women, SLE patients are at increased risk for maternal and fetal complications [14, 15]. According to the literature, one third of pregnancies will result in a cesarean section, 33\% will have preterm birth, and more than $20 \%$ will be complicated by preeclampsia [3,
4, 12, 16]. Pregnancy outcome in SLE and the risks for these complications relate to lupus activity, antiphospholipid antibodies, renal status, hypertension, and medication complications [4, 10, 13, 15].

The vast majority of our population was in remission prior to pregnancy and kept their medication throughout the gestation. The apparent low incidence of lupus flares in our population might be, at least partially, attributed to these aspects.

Antiphospholipid antibodies were present in 29\% of patients identical to most SLE series [6, 7]. All of these received aspirin and heparin. This approach is still controversial in the absence of clinical criteria for antiphospholipid syndrome [8]. From our experience, this therapy is not associated with worse maternal or fetal outcome namely bleeding disorders or osteoporosis reported in the literature [17]. In our population, aPL positivity was associated with more first-trimester miscarriages with no significant differences in maternal and perinatal parameters such as medical complications, FGR, gestational age at delivery, preterm delivery rate, cesarean section rate, and APGAR score.

Renal lupus accounted for $28 \%$ of cases but was responsible for $80 \%$ of the flares which highlights renal lupus as a major risk factor for adverse events in pregnancy $[14,15,18]$.

Hypertension and FGR were the most common complications, as expected. There was no clear relation between SLE activity or aPL and these events.

Our preterm delivery rate $(25 \%)$ was similar to the published data $[3,4]$.

Cesarean section was performed in $45 \%$ of patients, a figure higher than the published data although the main indications were related to fetal distress and not for maternal indication. One possible reason for this is the lower compliance of clinicians to signs of fetal distress in SLE "risky" patients.

Long-term effects of lupus in the offspring of SLE women are rare but include congenital heart block which can ultimately lead to the implantation of a definite pacemaker [19-23]. Positive SSa/SSbs are surrogates to this complication and all SLE patients should be screened for its presence. While most centers recommend a fetal echocardiogram every 4 weeks after 16 weeks gestation, our policy is to make an initial evaluation at 24 weeks gestation, with no need for further investigation if normal. Despite a high incidence of positive $\mathrm{SSa} / \mathrm{SSb}$ patients (nearly 50\%), there were no cases of congenital heart block in our series.

In conclusion, once considered a relative contraindication for pregnancy, SLE is no longer a reason for women not to get pregnant assuring that "safety" measures are taken and the expected course of pregnancy is fully 
explained to the patient, which is a major active participant in the process. Given the potential complications and morbidity, all pregnant women with SLE should be considered high risk and managed by a multidisciplinary team, ideally including a rheumatologist, an obstetrician with experience in lupus, an internist and, if indicated, a nephrologist [1].

Our autoimmune pregnancy clinic gathers the former conditions, with experienced obstetricians, an internal medicine specialist, and an excellent relationship with the other specialties. The majority of pregnancies in women with SLE in our series was successful and although associated with higher rates of prematurity and cesarean section than in normal subjects, these results are globally positive.

\section{References}

1. Witter F (2007) Management of the high-risk lupus pregnant patient. Rheum Dis Clin N Am 33:253-265

2. Clark C, Spitzer K, Laskin C (2005) Decrease in pregnancy loss rates in patients with systemic lupus erythematosus over a 40 year period. J Rheum 32(9):1709-1712

3. Clowse M, Jamison M, Myers E, James A (2008) A national study of the complications of lupus in pregnancy. Am J Obstet Gynecol 199:127.e1-127.e6

4. Clowse M (2007) Lupus activity in pregnancy. Rheum Dis Clin N Am 33:237-252

5. Gayed M, Gordon C (2007) Pregnancy and rheumatic diseases. Rheum 46:1634-1640

6. Irastorza G, Khamashta M (2007) Antiphopholipid syndrome in pregnancy. Rheum Dis Clin N Am 33:287-297

7. Petri M, Qazi U (2006) Management of antiphospholipid syndrome in pregnancy. Rheum Dis Clin N Am 32:591-607

8. Wilson WA, Gharavi AE, Piette JC (2001) International classification criteria for antiphospholipid syndrome. Lupus 10:457-460
9. Lockshin MD, Reinitz E, Druzin ML et al (1984) Lupus pregnancy. Case-control prospective study demonstrating absence of lupus exacerbation during or after pregnancy. Am J Med 77:893

10. Petri M, Howard D, Repke J (1991) Frequency of lupus flare in pregnancy (1991) The Hopkins Lupus Pregnancy Center experience. Arthritis Rheum 34:1538-1545

11. Yasmeen S, Wilkins E, Field N et al (2001) Pregnancy outcomes in women with systemic lupus erythematosus. J Mat Fet Med 10:91-96

12. Buyon JP (2005) Dispelling the preconceived notion that lupus pregnancies result in poor outcomes. J Rheumatol 32:1641-1642

13. Urowitz M, Gladman D, Farewell V et al (1993) Lupus and pregnancy studies. Arth Rheum 36(10):1392-1397

14. Johnson M, Petri M, Witter F et al (1995) Evaluation of preterm delivery in a systemic lupus erythematosus pregnancy clinic. Obst Gynecol 86:396-399

15. Petri M (2004) Prospective study of systemic lupus erythematosus pregnancies. Lupus 13(9):688-689

16. Clowse M, Witter FR, Magder LC, Petri M (2005) The impact of increased lupus activity on obstetrical outcomes. Arthritis Rheum 52:514-521

17. Cortes J, Ordi J, Paredes F et al (2002) Clinical predictors of fetal and maternal outcome in systemic lupus erythematosus: a prospective study of 102 pregnancies. Rheum (Oxford) 41(6):643650

18. Deruelle P, Coulon C (2007) The use of low-molecular-weight heparins in pregnancy-how safe are they? Curr Opin Obstet Gynecol 19:573-577

19. Moroni G, Ponticelli C (2003) The risk of pregnancy in patients with lupus nephritis. J Nephrol 16:161-167

20. Affleck AG (2006) Neonatal lupus erythematosus is not always benign. BMJ 332(7544):796

21. Tincani A, Rebaioli CB, Frassi M et al (2005) Pregnancy and autoimmunity: maternal treatment and maternal disease influence on pregnancy outcome. Autoimmun Rev 4(7):423-428

22. Brucato A, Frassi M, Franceschini F et al (2001) Risk of congenital complete heart block in newborns of mothers with anti-Ro/SSA antibodies detected by counterimmunoelectrophoresis: a prospective study of 100 women. Arthritis Rheum 44(8):1832-1835

23. Scott J (2002) Risks to the children born to mothers with autoimmune diseases. Lupus 11:655-660 